\title{
Morphological investigation of Cellulose Acetate nanofibrous membranes
}

\author{
Nasrin Attari ${ }^{1,2}$, Robert Hausler ${ }^{1,2}$, \\ ${ }^{1}$ École de Technologie Supérieure/Université du Québec \\ ${ }^{2}$ La Station Expérimentale des Procédés Pilotes en Environnement (STEPPE-ÉTS) \\ 1100 Notre-Dame St. W, Montreal, Québec, Canada \\ Nasrin.Attari.1@ens.etsmtl.ca; Robert.Hausler@etsmtl.ca
}

\begin{abstract}
In this work, we study the morphological changes in Cellulose Acetate (CA) electrospun nanofibrous membranes (ENMs) as a function of material parameters i.e. composition of the solvent and the concentration of the polymer, and electrospinning process parameters i.e. spinning solution flow rate, tip to collector distance (TCD), voltage field, and electrospinning processing time. In this respect, we have synthesized ten ENM samples using four different polymeric solutions that contained two concentrations at 10 wt.\% and $15 \mathrm{wt} . \%$ of cellulose acetate as the main polymer and three different volume ratios of N,N-dimethylformamide (DMF) and acetone mixtures $(1: 0,1: 1$, and 2:8) as solvent. The results show that the polymer solution with lower CA concentration forms thinner nanofibers at the same electrospinning process conditions. Moreover, by increasing the volume fraction of the DMF from $20 \mathrm{v} / \mathrm{v} \%$ to $100 \mathrm{v} / \mathrm{v} \%$ in the solvent, beads form with high density. We show that the solvent composition plays an important role in the formation of smooth and bead free nanofibrous membrane with high interconnective porosity. Furthermore, the electrospinning parameters imply that they affect the morphology of the ENMs significantly and further optimization of these parameters determines the ultimate morphology and volume fraction of nanofibers.
\end{abstract}

Keywords: Nanofibrous membranes, Nanostructured morphology, Cellulose acetate, Electrospinning

\section{Introduction}

Cellulose acetate (CA) is one of the most abundant and well-known polymer resources which has been widely used in the development of environmentally friendly membranes. CA membrane is a promising method for separation processes because of its numerous interesting characteristics. In general, CA polymer shows a combination of stimulating characteristics, such as high biodegradability, high hydrophilicity, nontoxicity, hardness, excellent chemical resistance, thermal stability, and relatively low cost (1-4). The extensive application of cellulose acetate membranes in nanofiltration processes has gotten the attention of scientists to the nanofibrous cellulose acetate due to its high surface to volume ratio, high interconnective porosity, and good chance to improve its mechanical strength (5).

On the other hand, electrospinning is proposed as a simple yet adjustable method where polymer solution is stretched using electrostatic forces against the surface tension of the spinning solution to fabricate electrospun nanofibrous membranes (ENMs) (6,7). Recently, ENMs have been become a promising alternative to be utilized in various applications such as membrane filtration, pharmaceutical industry, textile, food packaging, and tissue engineering. In this technique, which is based on the electric field between polymer solution droplets in the tip of the needle and the collector, the conicalshaped droplets are stretched out and form the nanofibers on the collector. Previous researchers suggest that several electrospinning process and material parameters e.g. TCD, feed flow rate, dope solution physical properties, voltage, and electrospinning processing time could affect the morphological characteristics of the membrane, including the size of pores, and their interconnectivity. Likewise, the composition of the dope solution affects the physical characteristics like viscosity, conductivity, surface tension, and volatility of the solution significantly $[1,8,9]$.

The main objective of this study is the analysis of morphological changes in CA ENMs and the impact of material and process parameters on it. In this respect, ten ENM samples were synthesized using four different polymeric solutions that contain CA as the main polymer with three different compositions of DMF and acetone as the solvent. The synthesis was performed by electrospinning and the influence of several electrospinning process parameters such as TCD, voltage power, electrospinning processing time, and feed flow rate were studied on morphological properties of nanofibrous cellulose acetate membrane samples. Furthermore, the impacts of solvent composition and polymer concentration in the dope solution on the porosity amount and the structure of ENMs were investigated. In section 2, we describe the materials and the approach we used. The results and discussion over the impact of the parameters are provided in section 3. 


\section{Materials and methods}

\subsection{Materials}

Cellulose Acetate $\left(\mathrm{CA}, \mathrm{M}_{\mathrm{n}}=50000, \rho=1.3 \mathrm{~g} / \mathrm{mL}\right)$ was purchased from Sigma-Aldrich Chemistry and used as the main polymer in this study. Different ratios of N, N-Dimethylformamide, and acetone that were obtained from Fisher Chemical Company were used as the solvent.

\subsection{Preparation and characterization of the polymer solution}

CA solutions at two concentrations of $10 \mathrm{wt} \%$ and $15 \mathrm{wt} . \%$ were prepared by dissolving powder CA polymer in three different solvent mixtures of DMF/acetone at ratios of (1:0), (1:1), and (2:8) by a magnetic stirrer for $24 \mathrm{~h}$ at 300 $\mathrm{rpm}$ at ambient temperature until the polymer was homogenously dissolved.

The viscosity of the solutions was determined by a digital viscometer (Brookfield) in a $20 \mathrm{~mL}$ cylindrical sample container at a constant solution temperature of $25^{\circ} \mathrm{C}$ using S-31 spindle. The rotation frequency of the S-31 spindle was set to $50 \mathrm{rpm}$ and the shear rate was $10.2 \mathrm{~s}^{-1}$.

The electrical conductivity of the spinning solutions was measured by an electrical conductivity meter (OAKLON $\mathrm{pH} / \mathrm{CON} 510$ Benchtop Meter) at a constant solution temperature of $25^{\circ} \mathrm{C}$. Table 1 summarizes the solutions, their compositions and the respective characteristics of these solutions.

Table 1: The properties and the characterization of the four dope solutions

\begin{tabular}{|c|c|c|c|c|}
\hline \multirow{2}{*}{ Solution \# } & CA Conc. ( & \multirow{2}{*}{$\begin{array}{c}\text { Solvent Comp. } \\
\text { wt. \%) }\end{array}$} & \multicolumn{2}{|c|}{ Solution Characterization } \\
\cline { 4 - 5 } & 10 & $1 / 0$ & 1812 & 7.70 \\
\hline 1 & 10 & $1 / 1$ & 827.8 & 6.38 \\
\hline 2 & 10 & $2 / 8$ & 664 & 6.08 \\
\hline 3 & 15 & $1 / 1$ & 1370 & 6.72 \\
\hline 4 & \multicolumn{2}{|c}{} \\
\hline
\end{tabular}

\subsection{Preparation of CA ENMs}

Figure 1 illustrates an schematic of the electrospinning technique used to fabricate nanofibrous membranes by preparation of homogeneous CA dope solutions. The set-up consists of a $20 \mathrm{~mL}$ BD plastic syringe as the solution container which is connected to a nozzle with inner diameter of $0.8 \mathrm{~mm}$ to form the fibers. A pump controls the feed rate of the solution. The produced nanofiber samples are collected using a collector that is covered by aluminum foil to facilitate the peeling off the membrane from the collector. Furthermore, a power supplier $(0-40 \mathrm{kV})$ is used to make the spinneret and the collector as two electrodes to provide electrostatic force to form the nanofibrous membrane mats.

The principle aim of the investigation of several electrospinning parameters such as polymer solution flow rate $(\mathrm{mL} / \mathrm{h})$, TCD $(\mathrm{mm})$, electric voltage field $(\mathrm{kV})$, and the electrospinning processing time $(\mathrm{h})$ was to achieve bead free nanofibrous membranes by the fabrication and characterizing of ten electrospun nanofibrous membrane samples. The electrospinning conditions and polymer solutions are summarized in Table 2.

\subsection{Characterization of CA nanofibrous membranes}

The morphology of CA ENMs was investigated by a Scanning Electron Microscope (SEM, Hitachi Model S3600$\mathrm{N})$. The SEM were performed at $5 \mathrm{kV}$ in three magnifications. The ENM samples were prepared for SEM study by coating their surface with a thin layer of gold using a sputter coater (Quorum Technologies - Model K550X). The coating process was performed in $35 \mathrm{~mA}$ for 2 minutes.

The porosity $(\varepsilon)$ of CA ENMs was measured by the deviation of the inter-fiber volume and the total volume of the membrane. For porous hydrophilic ENMs, it could be determined by a gravimetric method using Deionized water. The weighted dry membrane samples immersed in DI water overnight. The porosity was calculated by measuring the weight of water absorbed in the inter-fiber volume of the ENMs (3): 


$$
\varepsilon(\%)=\frac{\frac{\left(W_{w}-W_{d}\right)}{\rho_{w}}}{\frac{\left(W_{w}-W_{d}\right)}{\rho_{w}}+\frac{W_{d}}{\rho_{p}}} \times 100
$$

where $\mathrm{W}_{\mathrm{w}}$ is the weight of the wet sample $(\mathrm{g}), \mathrm{W}_{\mathrm{d}}$ is the dry sample weight $(\mathrm{g}), \rho_{\mathrm{p}}$ and $\rho_{\mathrm{w}}$ are the density of the water and polymer at $25^{\circ} \mathrm{C}$, respectively $\left(\mathrm{g} \cdot \mathrm{cm}^{-3}\right)$.

Table 2: The electrospinning process conditions for the ten samples.

\begin{tabular}{|c|c|c|c|c|c|c|c|}
\hline Samples\# & Solution\# & $\begin{array}{c}\text { Feed rate } \\
(\mathrm{ml} / \mathrm{h})\end{array}$ & $\begin{array}{c}\text { Voltage } \\
(\mathrm{kv})\end{array}$ & $\mathrm{TCD}(\mathrm{mm})$ & $\begin{array}{c}\text { Electrospinning } \\
\text { duration }\end{array}$ & $\begin{array}{c}\text { Temperature } \\
\left({ }^{\circ} \mathrm{C}\right)\end{array}$ & $\begin{array}{c}\text { Humidity } \\
(\%)\end{array}$ \\
\hline ENM 1 & 1 & 10 & 35 & 150 & 1 & 25.4 & 60 \\
\hline ENM 2 & 1 & 10 & 35 & 150 & 1 & 25.4 & 60 \\
\hline ENM 3 & 1 & 10 & 35 & 150 & 1 & 25.4 & 60 \\
\hline ENM 4 & 1 & 10 & 35 & 150 & 1 & 25.4 & 60 \\
\hline ENM 5 & 3 & 10 & 35 & 150 & 1 & 25.4 & 60 \\
\hline ENM 6 & 3 & 5 & 45 & 150 & 1 & 25.4 & 60 \\
\hline ENM 7 & 2 & 2 & 25 & 150 & 1 & 25.4 & 60 \\
\hline ENM 8 & 2 & 2 & 25 & 150 & 2 & 25.4 & 60 \\
\hline ENM 9 & 4 & 2 & 25 & 150 & 2 & 25.4 & 60 \\
\hline ENM 10 & 4 & 2 & 25 & 100 & 2 & 25.4 & 60 \\
\hline
\end{tabular}

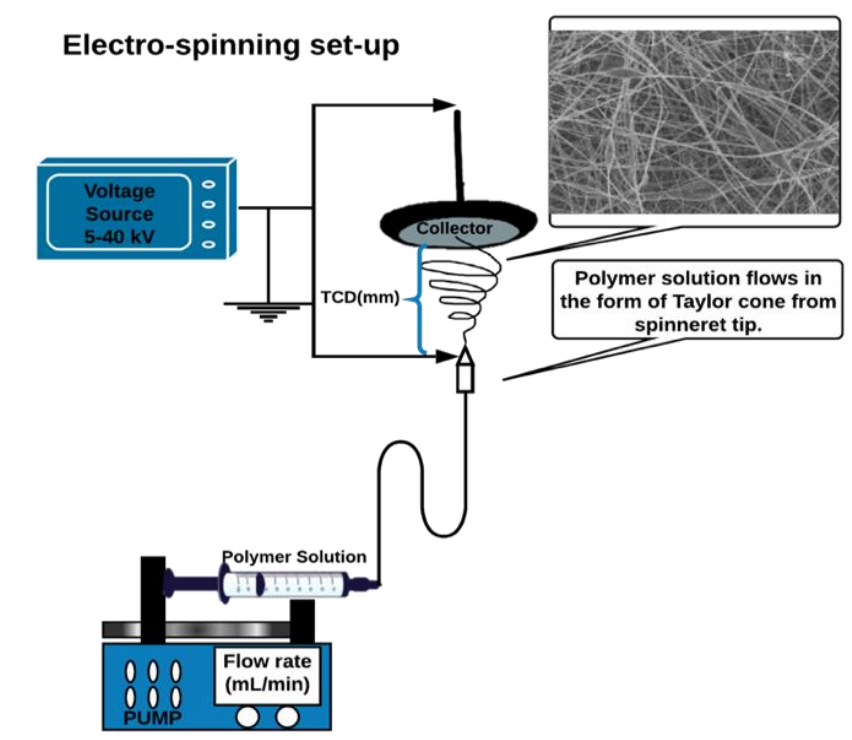

Fig. 1: Schematic diagram of the electrospinning technique.

\section{Results and discussions}

In this study, ten different CA membranes were fabricated in the laboratory by electrospinning method. We investigate the role of material and electrospinning parameters on the morphology and nanostructure of ENMs. The SEM, optical microscopy, and the porosity measurements were utilized to study the effect of the polymer concentration, composition of solvent, spinning solution flow rate, voltage field, TCD, and electrospinning processing time on the structure of the CA ENMs. 
Table 1 summarizes the characteristics of four spinning solutions that were prepared with different polymer concentrations and solvent compositions for the fabrication of CA ENMs. In this respect, the high volume fraction of DMF in the solvent and high concentration of CA result in the rise of the viscosity. Additionally, the conductivity of solutions depends on the DMF volume fraction in the solvent. By increasing the volume fraction of DMF in the solvent, the conductivity of the solution increases. The results show that the viscosity and the electrical conductivity of the spinning solutions is strongly correlated with the fiber formation process. Hence, we track the value of these parameters for all solutions prepared in this study.

\subsection{The effects of CA concentration and solvent composition in dope solution}

Figure 2 shows the SEM micrographs of the CA ENMs that was produced by electrospinning of three polymeric solutions with two CA concentrations of $10 \mathrm{wt} . \%$ and $15 \mathrm{wt} . \%$ and different solvent compositions with volume ratios of DMF to acetone as 1:1 and 2:8 that were contained 50 and $20 \mathrm{v} / \mathrm{v} \%$ DMF solvent respectively. As shown in Fig. 2(a, c), by increasing of CA concentration, the bead formation is declined. Also, according to Fig. 2(a) and Fig. 2(b), the low volume fraction of DMF in the solvent improves fiber formation. Hence, the solution properties, including polymer concentration and solvent quality have significant effects on the electrospinning process and the optimization of these parameters is an efficient way to control the structure of nanofibers $(1,10,11)$. The vital impact of solvent parameters like conductivity, solubility parameter, shear viscosity, surface tension, and concentration on ENMs structure is unavoidable and must be considered $(1,12-14)$.

The SEM micrograph shown in Fig. 2(a) indicates that a nanofibrous-structured membrane does not form when polymer concentration is low. This is due to the fact that at low concentration of polymer, the viscosity and surface tension remain low and the solution does not have a chance to stretch enough to form the nanofibrous-structured membranes.

Figure 2(b) shows the interaction between the polymer concentration and solvent composition parameters. The comparison of the Fig 2(a) and Fig 2(b) confirms that at constant polymer concentration, the decreasing of the DMF volume ratio from $50 \mathrm{v} / \mathrm{v} \%$ to $20 \mathrm{v} / \mathrm{v} \%$ in the solvent increases the surface tension and then results in nanofiber formation.

Figure 2(c) reveals that there has been a marked rise in the nanofiber formation by increasing the polymer concentration. It is notable that by increasing the polymer concentration from 10 wt.\% to 15 wt.\% which was dissolved in a solvent with $50 \mathrm{v} / \mathrm{v} \% \mathrm{DMF}$, the polymer concentration effect could prevail over the high DMF volume fraction in the solvent and results in to form smooth and bead-free nanofibrous structure.

\subsection{The effects of electrospinning parameters}

The SEM micrographs of six ENM samples can be compared to investigate the morphological effects of electrospinning parameters i.e. the applied voltage field, the feed flow rate, the processing time, and the TCD. The structural impact of applied voltage field on the ENMs has been reported in Fig. 3(a) and Fig. 3(b). It is shown that by increasing the voltage field from $35 \mathrm{kV}$ to $45 \mathrm{kV}$, the fibers became thinner, and the polymer accumulation and bead formation are declined due to the higher electrostatic force between two electrodes, which causes the fibers to stretch more into thinner size (15).

Furthermore, it can be seen from Fig. 3(a) and Fig. 3(b) that by decreasing the polymer solution flow rate from 10 $\mathrm{ml} / \mathrm{h}$ to $5 \mathrm{ml} / \mathrm{h}$, the thinner fibers were formed, and there was less accumulation of polymer solution in electrospun membrane mat.

According to Fig. 3(c) and Fig. 3(d), the density of beads per surface unit of the ENM was increased due to the more processing time in ENM sample number 8.

Fig. 3(e) and Fig. 3(f) presents the micrographs of ENM numbers 9 and 10 samples, which were fabricated in different TCDs of $150 \mathrm{~mm}$ and $100 \mathrm{~mm}$, respectively. Since TCD could affect the strength of the electrostatic force and also the evaporation rate of residual solvent in the stretched fiber, the longer TDC causes thinner fiber size, which is in accordance with the represented micrographs in Fig. 3(e, f) (16). 
All results reported in this section are in consistent with the morphological impact of the electrical conductivity of the spinning solutions, which are reported in Table 1. The increasing of the electrical conductivity of the spinning solution causes to high charge repulsion and elongation during the electrospinning process. Hence, in the same processing conditions, the spinning of the more conductive solution forms thinner fibers in the ENM structure (17).

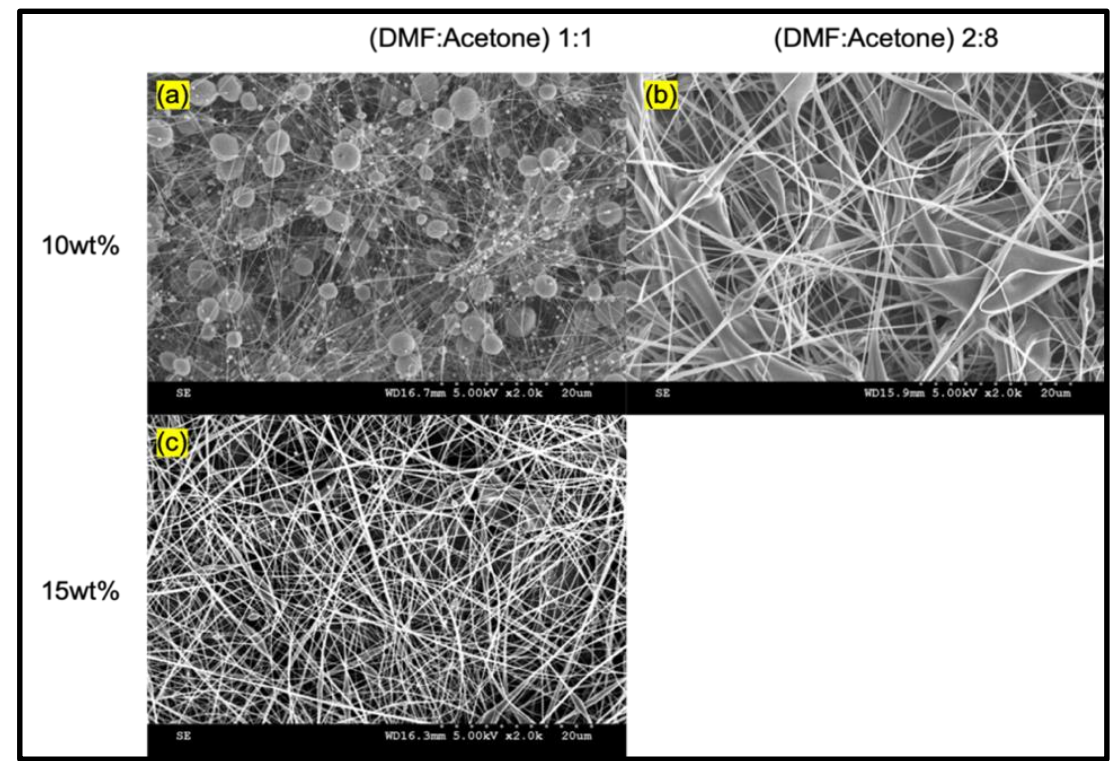

Fig. 2: SEM micrographs of ENM samples. Sample number a) 7, b) 6, and c) 9. The morphological effects of CA concentration and solvent composition are evident.

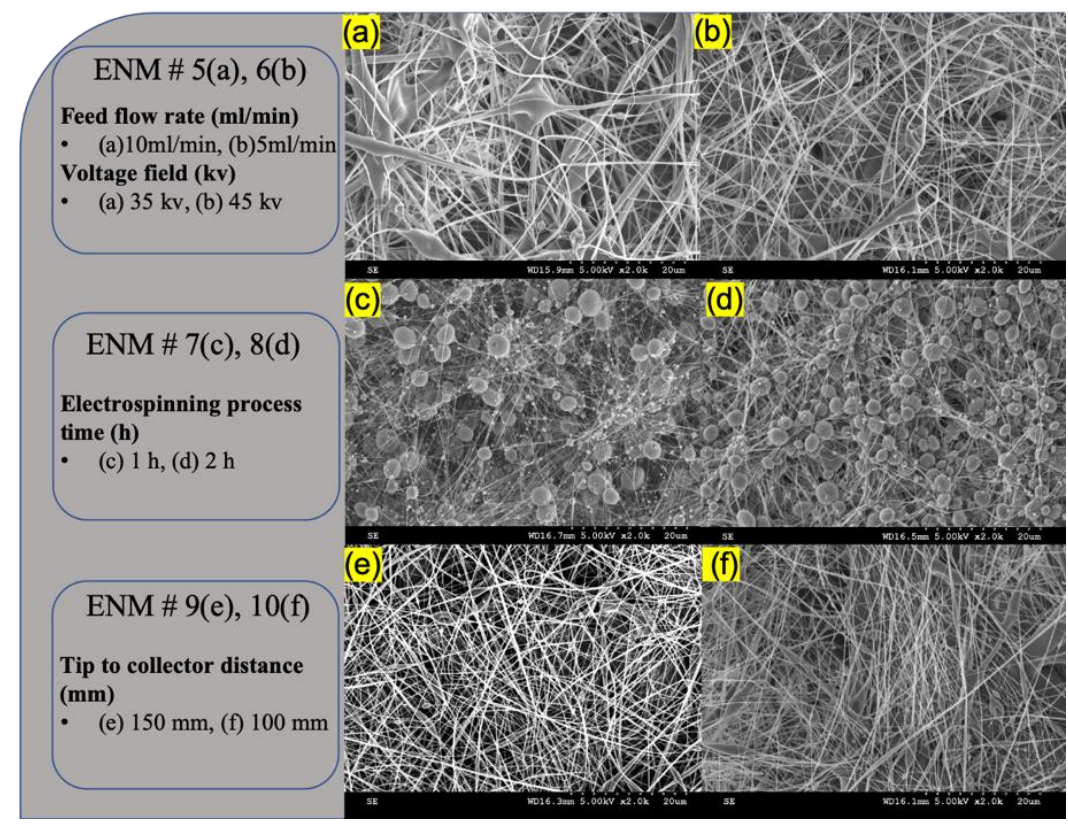

Fig. 3: SEM micrographs of ENM samples. Sample number a) 5, b) 6, c) 7, d) 8, e) 9, and f) 10. The effects of feed flow rate, voltage, processing time, and TCD on the ENMs structure are evident. 


\subsection{The effects of material and electrospinning parameters on the porosity of CA ENMs}

Porosity ( $\varepsilon$ ) amount of electrospun nanofibrous membranes is one of the fundamental characteristics which plays a prominent role in their efficiency during a separation process. The nanofibers with the small pore size and high interconnective porosity could filtrate water selectively. Hence, in this section, the effect of material and electrospinning parameters on porosity amount of the ENMs were studied. The results of porosity measurement analysis are summarized in the Fig. 4 and Fig. 5.

It can be seen from Fig. 4 that the porosity amount of the ENMs is raised from $58 \%$ to $81 \%$ by decreasing the DMF volume ratio from $100 \mathrm{v} / \mathrm{v} \%$ to $20 \mathrm{v} / \mathrm{v} \%$. As we discussed in section 3.2, low volume fraction of DMF in the solvent suppress the bead formation and cause to increase porosity amount in the CA ENMs.

Figure 5 provides the results obtained for the effect of CA concentration on porosity amount of ENMs. The increasing of CA concentration from $10 \mathrm{wt} . \%$ to $15 \mathrm{wt} . \%$ results in a remarkable rise in porosity from $82 \%$ to 97 $\%$. Due to the fact that bead formation in the membrane structure causes pore blockage and since, by increasing the CA concentration in spinning solution the bead formation is suppressed, the porosity of ENM sample is improved by increasing of CA concentration in the spinning solution.

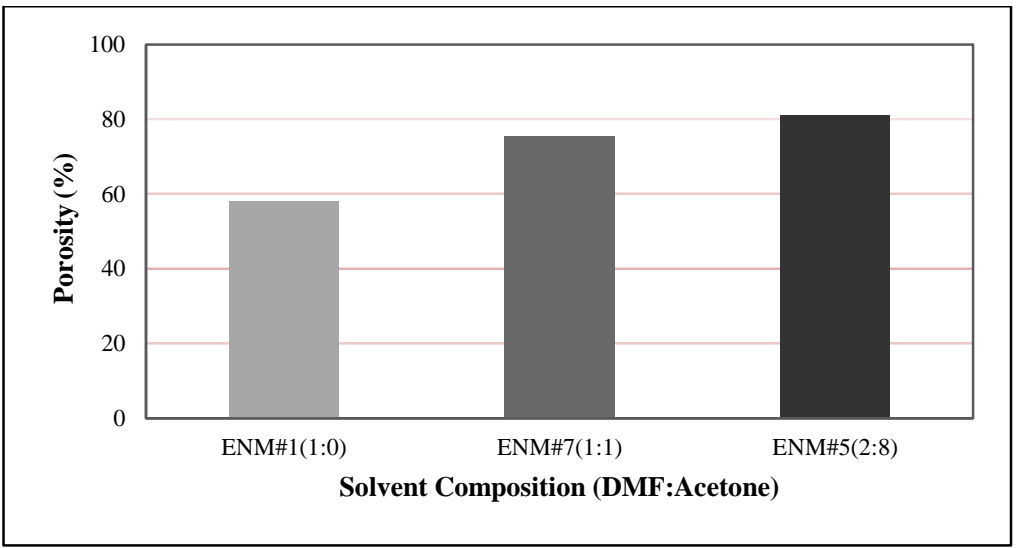

Fig. 4: The porosity amounts ( $($ ) of ENM\# 1,5, and 7 samples, which are synthesized of different solvent compositions and the same CA concentration of $10 \mathrm{wt} . \%$ in the constant electrospinning conditions.

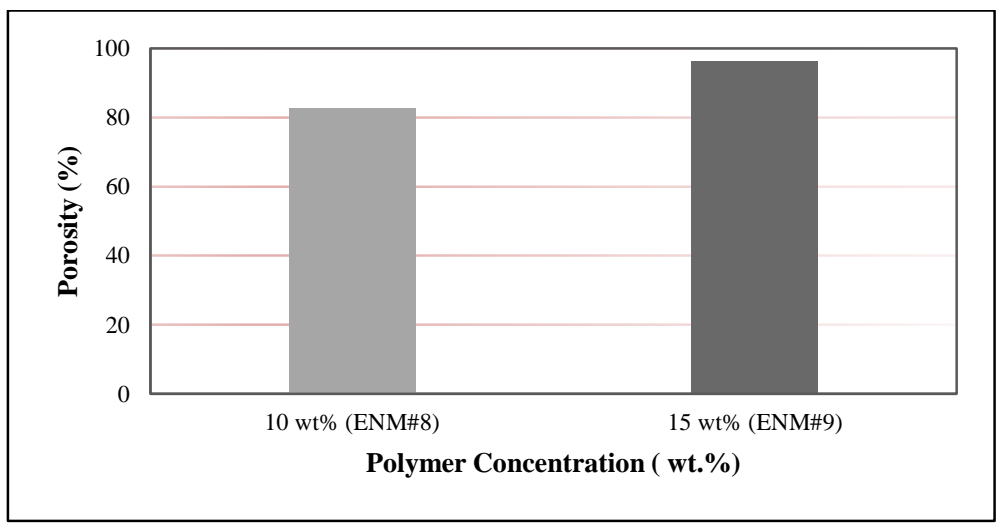

Fig. 5: Porosity amounts ( $\varepsilon$ ) of ENM\# 8 and 9 samples, which are synthesized of different CA concentrations of 10 wt.\% and 15 wt.\% and the same volume fraction of DMF/Acetone solvents (1:1) in the constant electrospinning conditions. 
Figure 6 provides the porosity amounts of eight CA ENM samples to investigate the effect of electrospinning parameters on the porosity of ENMs. It can be seen from Fig. 6 that by increasing the feed flow rate from $5 \mathrm{ml} / \mathrm{h}$ to $10 \mathrm{ml} / \mathrm{h}$ in ENM samples number 5 and 6 due to the high formation rate of fibers and less bead presence in the structure of the membrane, the porosity amount was raised. Additionally, by increasing the applied voltage field from $35 \mathrm{kV}$ to $45 \mathrm{kV}$, the bead formation is suppressed and as a result, the porosity was increased. As discussed before in section 3.2, by decreasing the distance between spinneret tip and the collector from $150 \mathrm{~mm}$ to $100 \mathrm{~mm}$ by synthesizing ENM samples number 9 and 10 samples, more beads and polymer accumulation in the membrane structure were occurred, and consequently, porosity amount was declined. The reason for the porosity improvement due to the rise in electrospinning process time from $1 \mathrm{~h}$ to $2 \mathrm{~h}$ by fabrication of ENM samples number 7 and 8 samples is the increasing of the fiber formation which is confirmed by porosity test as well.

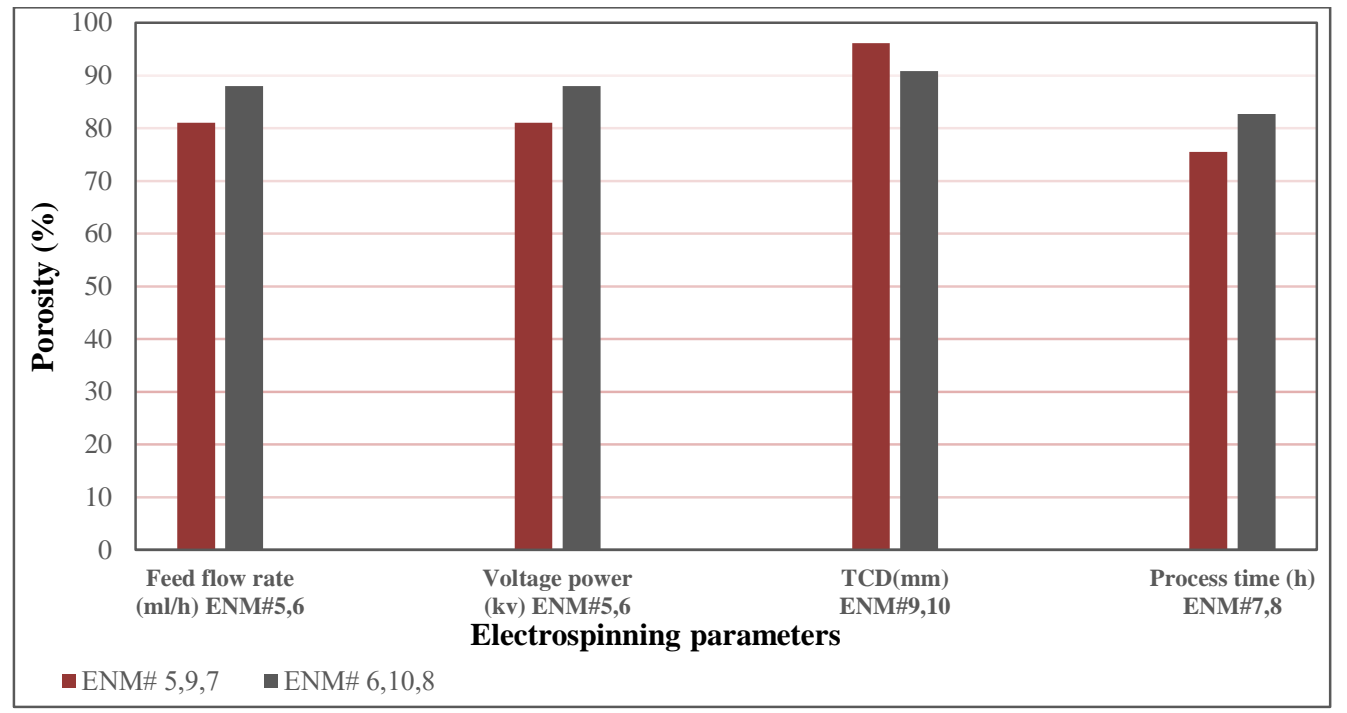

Fig. 6: Porosity amounts $(\varepsilon)$ of ENM\# $(5,6),(7,8)$, and $(9,10)$ samples to study the effect of feed flow rate, voltage field, TCD, and process time parameters. The material and electrospinning properties of all samples are presented in Table 2.

\section{Conclusion}

We report the effect of two material parameters, CA concentration and solvent composition in the spinning solution, and electrospinning parameters, feed flow rate, TCD, voltage power, and processing time on the nanostructure of fabricated CA ENMs from the morphological point of view. This study has identified that the preparation of a dope solution using higher CA concentration and lower DMF volume fraction in the solvent mixture improves the fibrous structure of the ENMs and also results in a more interconnective porosity. Additionally, investigation of the effects of four electrospinning parameters will prove useful in expanding our understanding of efficient synthesize of ENMs for high quality water purification applications.

\section{Acknowledgments}

Authors sincerely acknowledge the financial support from the Arbour Foundation of Montreal, Quebec, Canada. Authors also gratefully appreciate Biointerface Research Center of McGill University and Dr. Marta Cerruti, the head of the center, for providing electrospinning apparatus. 


\section{References}

[1] Lee H, Nishino M, Sohn D, Lee JS, Kim IS. Control of the morphology of cellulose acetate nanofibers via electrospinning. Cellulose. 2018;25(5):2829-37.

[2] Battirola LC, Andrade PF, Marson GV, Hubinger MD, do Carmo Gonçalves M. Cellulose acetate/cellulose nanofiber membranes for whey and fruit juice microfiltration. Cellulose. 2017;24(12):5593-604.

[3] Etemadi H, Yegani R, Babaeipour V. Performance evaluation and antifouling analyses of cellulose acetate/nanodiamond nanocomposite membranes in water treatment. J Appl Polym Sci. 2017;134(21).

[4] Chiţanu E, Băra A, Banciu C, Lungulescu M, Marinescu V. Study of electrospun cellulose acetate fibers. Ind Textila. 2018;69(5):363-8.

[5] Ma H, Burger C, Hsiao BS, Chu B. Fabrication and characterization of cellulose nanofiber based thin-film nanofibrous composite membranes. J Membr Sci. 2014;454:272-82.

[6] Aruchamy K, Mahto A, Nataraj SK. Electrospun nanofibers, nanocomposites and characterization of art: Insight on establishing fibers as product. Nano-Struct Nano-Objects. 2018;16:45-58.

[7] Pan C-Y, Xu G-R, Xu K, Zhao H-L, Wu Y-Q, Su H-C, et al. Electrospun nanofibrous membranes in membrane distillation: Recent developments and future perspectives. Sep Purif Technol. 2019;221:44-63.

[8] Drioli E, Giorno L, Fontananova E. Comprehensive membrane science and engineering. Second. Elsevier; 2017.

[9] Suja PS, Reshmi CR, Sagitha P, Sujith A. Electrospun nanofibrous membranes for water purification. Polym Rev. 2017;57(3):467-504.

[10] Liu H, Tang C. Electrospinning of cellulose acetate in solvent mixture N, N-dimethylacetamide (DMAc)/acetone. Polym J. 2007;39(1):65-72.

[11] Phan D-N, Lee H, Choi D, Kang C-Y, Im SS, Kim IS. Fabrication of two polyester nanofiber types containing the biobased monomer isosorbide: poly (ethylene glycol 1, 4-cyclohexane dimethylene isosorbide terephthalate) and poly (1, 4-cyclohexane dimethylene isosorbide terephthalate). Nanomaterials. 2018;8(2):56.

[12] Tungprapa S, Puangparn T, Weerasombut M, Jangchud I, Fakum P, Semongkhol S, et al. Electrospun cellulose acetate fibers: effect of solvent system on morphology and fiber diameter. Cellulose. 2007;14(6):563-75.

[13] Han SO, Youk JH, Min KD, Kang YO, Park WH. Electrospinning of cellulose acetate nanofibers using a mixed solvent of acetic acid/water: Effects of solvent composition on the fiber diameter. Mater Lett. 2008;62(4-5):759-62.

[14] Uyar T, Besenbacher F. Electrospinning of uniform polystyrene fibers: The effect of solvent conductivity. Polymer. 2008;49(24):5336-43.

[15] Sill TJ, von Recum HA. Electrospinning: applications in drug delivery and tissue engineering. Biomaterials. 2008;29(13):1989-2006.

[16] Matabola KP, Moutloali RM. The influence of electrospinning parameters on the morphology and diameter of poly (vinyledene fluoride) nanofibers-effect of sodium chloride. J Mater Sci. 2013;48(16):5475-82.

[17] Tijing LD, Woo YC, Yao M, Ren J, Shon HK. 1.16 Electrospinning for membrane fabrication: strategies and applications. Compr Membr Sci Eng 2nd Edn Elsevier Oxf. 2017;418-44. 